\title{
INTERNAL TRAINING LOAD AND FITNESS PROFILE BETWEEN ADULT TEAM VERSUS JUNIOR TEAM SOCCER PLAYERS
}

\author{
Alireza Rabbani ${ }^{1}$, Del P. Wong ${ }^{2}$, Filipe Manuel Clemente ${ }^{3,4}$, and Mehdi Kargarfard ${ }^{1}$ \\ ${ }^{I}$ Department of Exercise Physiology, Faculty of Sport Sciences University of Isfahan, Iran \\ ${ }^{2}$ School of Nursing and Health Studies, The Open University of Hong Kong, Hong Kong \\ ${ }^{3}$ Escola Superior Desporto e Lazer, Instituto Politécnico de Viana do Castelo, \\ Viana do Castelo, Portugal \\ ${ }^{4}$ Instituto de Telecomunicações, Delegação da Covilhã, Covilhã, Portugal
}

Original scientific paper

DOI: $10.26582 / \mathrm{k} .53 .1 .8$

\begin{abstract}
:
The aim of the present study was to compare the fitness profiles and internal training loads between senior team and academy team soccer players during an in-season phase. Twenty-two professional soccer players from the senior team ( $\mathrm{n}=12 ; 28.3 \pm 2.0$ years) and under 19 (U19) team ( $\mathrm{n}=10 ; 18.0 \pm 0.4$ years) of the same club participated in the present study. High-intensity running performance, acceleration, maximal sprint, and change of direction (COD) ability were all tested during the mid-season break of a competitive season. Session rating of perceived exertion (sRPE) reflecting the internal training load during the entire first half of the season was being documented daily. Senior players showed small to moderate superiority in $\operatorname{COD}(1.8 \%, 90 \%$ confidence intervals [CI, $-3.2 ; 7.1]$, ES: 0.24 [-0.44; 0.92]), maximal sprint (2.3\%, [0.0; 4.7], ES: 0.81 [0.00; 1.63]) and acceleration (3\%, [0.2; 5.8], ES: 0.96 [0.06; 1.85]). The U19 showed small better high-intensity intermittent running fitness $(2.5 \%$, [-1.2; 6.3], ES: 0.39 [-0.20; 0.97]). When analyzing internal training loads (from M-3 to M+3), the U19 showed small to very large higher sRPE values for all days (range; $8.2 \% ; 229.3 \%,[-8.1 ; 328.3]$, ES range; $0.25 ; 2.70,[-0.26 ; 3.3]$ ), except for match days $(\mathrm{M})$, on which unclear trivial difference was observed $(-1.5 \%$, [-9.6; 7.5], ES $-0.09[-0.65 ; 0.46])$. Our results showed that senior players and youth players had different fitness profiles and internal training loads during the first half of a competitive season; this should be taken into consideration when designing specific and individualized recovery and training sessions.
\end{abstract}

Key words: football, high-intensity intermittent performance, perceived exertion, change of direction, maximal sprint, acceleration

\section{Introduction}

Soccer is an intense team sport demanding players to have a high level of fitness to cope with the intermittent nature of match play (Bangsbo, 1994). A strong fitness profile not only reduces the risk of injury and increases the players' availability (Malone, et al., 2017; McCall, et al., 2014), but it also improves soccer performance (Helgerud, Engen, Wisloff, \& Hoff, 2001). Recently, an increasing trend of physical and technical performance has been documented in a high-level soccer. This may be, at least in part, pertinent with more intense training interventions (Barnes, Archer, Hogg, Bush, \& Bradley, 2014). Increases of 30, 35 and $80 \%$ have occurred during recent years in highintensity running distance, sprint distance, and number of sprints, respectively (Barnes, et al.,
2014), highlighting the important consideration of players' fitness conditions at higher levels of soccer (Bangsbo, Iaia, \& Krustrup, 2008; le Gall, Carling, Williams, \& Reilly, 2010; Metaxas, Koutlianos, Sendelides, \& Mandroukas, 2009).

The intense nature of high-level soccer (Bangsbo, 1994; Barnes, et al., 2014) and the current special fitness-developing interventions (e.g., high-intensity interval training) in soccer academies (Buchheit \& Rabbani, 2014) may have caused different fitness levels between various age categories (Al Haddad, Simpson, Buchheit, Di Salvo, \& Mendez-Villanueva, 2015; le Gall, et al., 2010). It seems that as the age of players increases, their fitness level is also enhanced (Al Haddad, et al., 2015; Bangsbo, et al., 2008; le Gall, et al., 2010) due to maturity and greater involvement in 
training and/or matches with (likely) greater intensities (Jaspers, Brink, Probst, Frencken, \& Helsen, 2016). The increasing fitness level trend from youth to adult age categories (Al Haddad, et al., 2015; Bangsbo, et al., 2008; le Gall, et al., 2010) as well as a more suitable fitness profile of players at higher levels of soccer (Al Haddad, et al., 2015; Bangsbo, et al., 2008; Djaoui, Chamari, Owen, \& Dellal, 2017; Metaxas, et al., 2009; Mujika, Santisteban, Impellizzeri, \& Castagna, 2009; Wong \& Wong, 2009) suggest that, in professional clubs, senior team players may show a higher level of fitness than their younger academy peers.

Despite the maturity-induced advantages acquired after the time of puberty by soccer players (le Gall, et al., 2010), the amount of imposed internal training load may also play an important role in fitness improvement (Campos-Vazquez, ToscanoBendala, Mora-Ferrera, \& Suarez-Arrones, 2017; Jaspers, et al., 2016). Campos-Vazquez et al. (2017) have recently shown that perceived accumulated internal training load (i.e., session rating of perceived exertion [sRPE]) is a determinant factor in the changes of high-intensity intermittent fitness in professional players. The large positive correlation $(r>0.7)$ observed between the accumulated SRPE and changes in the maximal speed reached during the last stage of a 30-15 Intermittent Fitness Test (30-15 $5_{\mathrm{IFT}}, \mathrm{V}_{\mathrm{IFT}}$ ) showed that internal training load could have paramount importance when monitoring training status of soccer players (CamposVazquez, et al., 2017). However, inconsistencies exist in the results of studies on the effect of training load indicators on neuromuscular fitness outcomes (Jaspers, et al., 2016).

Therefore, observing a greater daily internal training load, which is likely responsible for the superior fitness profile of players at higher levels of soccer, would be expected. To the best of our knowledge, however, there is no study which has compared the fitness characteristics and daily internal training loads of senior team players and academy team players over a prolonged period of time. Subsequently, the aim of the present study was to compare the fitness profiles and internal training loads of senior team and academy team soccer players during an in-season phase of annual periodization. We hypothesized that senior team players would present a better fitness profile and tolerate greater amounts of daily internal training loads during the in-season phase.

\section{Methods}

\section{Experimental approach}

In this study, we aimed to compare the fitness profiles and internal training loads of senior team and youth team soccer players from a single soccer club which competes in the Persian Gulf League of
Iran. Based on recent evidence of the dose-response relationship between fitness outcome and internal training loads in soccer players (Campos-Vazquez, et al., 2017; Jaspers, et al., 2016), we collected sRPE daily, as a valid training load measure (Impellizzeri, Rampinini, Coutts, Sassi, \& Marcora, 2004) during the first half (i.e., 15 weeks and 10 weeks for the senior team and youth team, respectively) of the competitive season. All players were also tested in terms of acceleration, maximal sprint (Djaoui, et al., 2017), change of direction ability-COD (Draper \& Lancaster, 1985), and high-intensity running performance (Buchheit, 2008) as important fitness factors in soccer (Bangsbo, 1994; Barnes, et al., 2014) during the mid-season, one week after the last match of the first half of the season. Acceleration, maximal sprint, and COD ability were all tested in the first testing session; 72 hours later, high-intensity intermittent running performance was assessed using 30-15 $5_{\mathrm{IFT}}$. A standardized 15-minute warm-up preceded both testing sessions, starting with five minutes of linear jogging. Five minutes of dynamic stretching emphasizing lower body limbs was then implemented. The last five minutes of warm-up consisted of progressive running and COD exercises, with rest intervals between actions to prevent the occurrence of a possible fatigue effect. All players from both teams were habituated to all maximal performance tests as they were part of their routine monitoring test battery.

\section{Participants}

Twenty-two professional players from the senior team $(n=12)$ and U19 team $(n=10)$ from the same soccer club competing in the Persian Gulf League of Iran participated in the present study (see Table 1). All participants were outfield players competing in the 2015-2016 season at the highest level in their respective age categories. These data were collected as a condition of player monitoring in which player activities were routinely measured over the course of the competitive season; therefore, ethics committee clearance was not required (Winter \& Maughan, 2009). However, a letter of informed consent was signed by all participants before they began the study. The experiment protocol conformed to the Helsinki Declaration.

\section{Data collection}

\section{Testing and training}

High-intensity running performance, acceleration, maximal sprint, and COD ability were all tested during the mid-season break using $30-15_{\text {IFT }}$ (Buchheit, 2008), 10-m acceleration, 30-m linear sprint, and 505 COD (Draper \& Lancaster, 1985) tests for important fitness characteristics of professional soccer players (Djaoui, et al., 2017; Paul \& Nassis, 2015). All tests were conducted on artifi- 
cial turf between 10 a.m. and noon, with a similar air temperature $\left(18-20^{\circ} \mathrm{C}\right)$. No official matches or intense workouts were carried out during 48 hours before the assessment. The internal training load during the entire first half of the season of the competitive year was documented daily. Data from the first day after the match $(\mathrm{M}+1)$, the second day after the match $(\mathrm{M}+2)$, and the third day after the match $(\mathrm{M}+3)$ for those players who had not played the match $(\mathrm{M})$ were eliminated from analyses to keep the conditions between both groups as similar as possible. Training protocols were similar for the U19 and senior team groups. The training protocol on the $\mathrm{M}+1$ day was designed to recover players from the match; it included lowto-moderate-intensity aerobic endurance exercises and recovery conditions. Training sessions on $\mathrm{M}+2$ consisted mostly of concurrent resistance and technical training. The protocols on $\mathrm{M}+3$ as well as on three days prior to the match day (M-3) included high-intensity neuromuscular or metabolic training, mostly using small-sided games (SSGs) (Hill-Haas, Dawson, Impellizzeri, \& Coutts, 2011). Two days and one day prior to the match day (M-2 and M-1, respectively) were low-load training days, intending to refresh players and to implement tapering strategies to have the maximum potential of players for the upcoming match day.

\section{High-intensity intermittent running performance measure}

The $30-15_{\text {IFT }}$ is a valid progressive field-based test designed to measure the high-intensity intermittent running performance of team athletes (Buchheit, 2008). In brief, the test consists of 30 -second runs interspersed with 15 -second recovery phases. The first running level of $30-15_{\text {IFT }}$ starts at $8 \mathrm{~km} \cdot \mathrm{h}^{-1}$ with $0.5 \mathrm{~km} \cdot \mathrm{h}^{-1}$ speed increments at each level. The length of the test field is $40 \mathrm{~m}$ but it is divided in the middle into two separate $20-\mathrm{m}$ parts. At both 40-m terminals and at the middle $20-\mathrm{m}$ line, $3-\mathrm{m}$ zones are created. Running velocity is adjusted with an auditory signal, and the subjects have to reach the 3-m zones when the alarm sounds. The subjects continue running until exhaustion, which is noted by the inability to continue with the imposed running speed and, subsequently, not being within the 3-m zones at the moment of the alarm for three consecutive sounds. The maximal speed reached during the last completed stage of the $30-15_{\mathrm{IFT}}\left(\mathrm{V}_{\mathrm{IFT}}\right)$ is considered the final performance.

\section{Measures of acceleration, maximal sprint, and change of direction}

Acceleration was assessed using a $10-\mathrm{m}$ sprint from a stationary start position. Maximal sprint was evaluated from a linear 30-m sprinting time. Change of direction ability was measured with a 505 test (Draper \& Lancaster, 1985). For the 505 test the players assumed a starting position $10 \mathrm{~m}$ away from the timing gate to reach a high level of acceleration. After completing a $15-\mathrm{m}$ linear sprint, players were instructed to turn (5 $\mathrm{m}$ away from the other part of the timing gate) with their preferred foot and to return to pass the timing gate again. For all the acceleration, linear sprint, and COD ability tests, the players were instructed to perform as quickly as possible for three trials with a 5-min recovery between sets; each player's best performance of the three was recorded.

\section{Measure of internal training load}

During all training and match sessions of the first half of the season, RPE and total minutes of training sessions were collected daily for each individual to calculate SRPE as the internal training load (Impellizzeri, et al., 2004). RPE (CR-10-point scale) was assessed 30 min after training sessions; the ratings were made individually. After that, the CR-10 rating was multiplied by the time of training, in minutes. The sRPE has been shown to have high validity as a measure of internal training load (Impellizzeri, et al., 2004), and, recently, its measurements have been shown to be sensitive to changes in intermittent fitness among professional soccer players (Campos-Vazquez, et al., 2017).

\section{Statistical analyses}

The results are presented as mean $\pm \mathrm{SD}$ and/ or $90 \%$ confidence intervals (CI). The average sRPE on all days (i.e., $\mathrm{M}-3, \mathrm{M}-2, \mathrm{M}-1, \mathrm{M}, \mathrm{M}+1$, $\mathrm{M}+2, \mathrm{M}+3$ ) were calculated for each individual. The pooled average of the sRPE of all training and match sessions was also calculated for each individual as a general index of all sessions' internal training loads. The differences between senior and U19 team players in terms of mean SRPE for each day and in terms of fitness variables were analyzed using magnitude-based inferences (MBI) and Cohen's $d$ effect size (ES) with 90\% CI (Hopkins, Marshall, Batterham, \& Hanin, 2009). Probabilities were also calculated to analyze whether the true difference was lower than, similar to, or higher than the smallest worthwhile difference (Hopkins, et al., 2009). If the probabilities of the effects being either substantially positive or negative were $>5 \%$, the effect was reported as unclear; otherwise, the effect was clear and reported as the magnitude of the observed value. The scale was as follows: $25-75 \%$ $=$ possible; $75-95 \%=$ likely; $95-99 \%=$ most likely; $>99 \%=$ almost certain (Hopkins, et al., 2009).

\section{Results}

Subjects' age and anthropometric characteristics are shown in Table 1. Descriptive information on fitness variables of the senior and U19 teams are presented in Table 2. The senior team players showed possibly-to-likely superiority (small to 
moderate magnitude) (range: $1.8 ; 3 \%, 90 \%$ confidence interval: [CI, -3.2; 7.1], ES range: 0.24; 0.96, $[-0.44 ; 1.85])$ in COD, maximal sprint and acceleration variables (Figure 1). A possibly higher (small magnitude) intermittent running performance $\left(\mathrm{V}_{\mathrm{IFT}}\right)$, however, was observed in the U19 range; $2.47 ; 2.70,[1.92 ; 3.03]$ ) (Figure $2 / \mathrm{C}$ ). When average sRPE for each individual was pooled from all training sessions, the U19 team players showed almost certainly greater (moderate magnitude) values than the senior team players $(21.7 \%$, [11.0; 33.4], ES 1.12 [0.59; 1.64]).

Table 1. Subjects' age and anthropometric characteristics

\begin{tabular}{lcccc}
\hline Group & Age (year) & Body mass $(\mathrm{kg})$ & Height $(\mathrm{m})$ & BMI $\left(\mathrm{kg} \cdot \mathrm{m}^{-2}\right)$ \\
\hline First team & $28.3(2.0)$ & $72.5(6.6)$ & $1.78(0.7)$ & $22.6(0.5)$ \\
U19 & $18.0(0.4)$ & $71.5(4.9)$ & $1.80(0.4)$ & $22.2(1.5)$ \\
\hline
\end{tabular}

Note: $\mathrm{BMI}$ - body mass index

Table 2. Fitness profile of subjects

\begin{tabular}{lcccc}
\hline Group & $\begin{array}{c}\text { Acceleration }(0-10 \mathrm{~m}) \\
\text { time }(\mathrm{s})\end{array}$ & $\begin{array}{c}\text { Maximal sprint }(0-30 \mathrm{~m}) \\
\text { time }(\mathrm{s})\end{array}$ & $\begin{array}{c}\text { COD } \\
\text { time }(\mathrm{s})\end{array}$ & $\begin{array}{c}\mathrm{V}_{\text {IFT }} \\
\left(\mathrm{km} \cdot \mathrm{h}^{-1}\right)\end{array}$ \\
\hline First team & $1.80(0.05)$ & $4.20(0.11)$ & $2.31(0.15)$ & $19.0(1.0)$ \\
U19 & $1.85(0.07)$ & $4.30(0.14)$ & $2.35(0.12)$ & $19.5(0.7)$ \\
Effect size (Cl) & $0.96(0.90)$ & $0.81(0.81)$ & $0.24(0.68)$ & $-0.39(0.58)$ \\
\hline
\end{tabular}

Note: $\mathrm{V}_{\mathrm{IFT}}$ - maximal velocity reached in the last stage of the 30-15 Intermittent Fitness Test; $\mathrm{Cl}$ - confidence interval; $\mathrm{COD}-$ changeof-direction

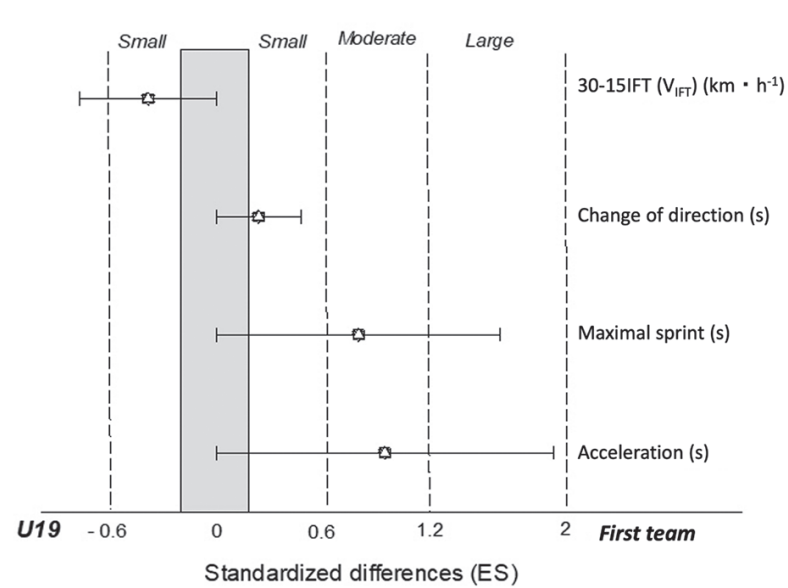

Note: ES - effect size; 30-15 $5_{\mathrm{IFT}}-30-15$ Intermittent Fitness Test; $\mathrm{V}_{\mathrm{IFT}}$ - maximal velocity reached during the last stage of $30-15_{\mathrm{IFT}}$.

Figure 1. Standardized differences (90\% confidence intervals) in fitness variables between the groups. Shaded area refers to the range of trivial difference (see Methods).

team $(2.5 \%,[-1.2 ; 6.3]$, ES $0.39[-0.2 ; 0.97])$ (Figure 1). When analyzing internal training load measures for each day (from M-3 to M+3), the U19 team showed likely-to-almost certainly higher (small to very large magnitude) $\mathrm{sRPE}$ for all days (range; 8.2 ; $229.3 \%$, [-8.1; 328.3], ES range; 0.25; 2.70, [-0.26; 3.3]) except for the match day (M), in which unclear trivial difference was observed $(-1.5 \%,[-9.6 ; 7.5]$, ES -0.09 [-0.65; 0.46]) (Figure 2). Almost certainly very large differences in SRPE were observed on the first and second days after the match (i.e., $\mathrm{M}+1, \mathrm{M}+2)$ (range; 105.5; 229.3\%, [-74.9; 328.3], ES

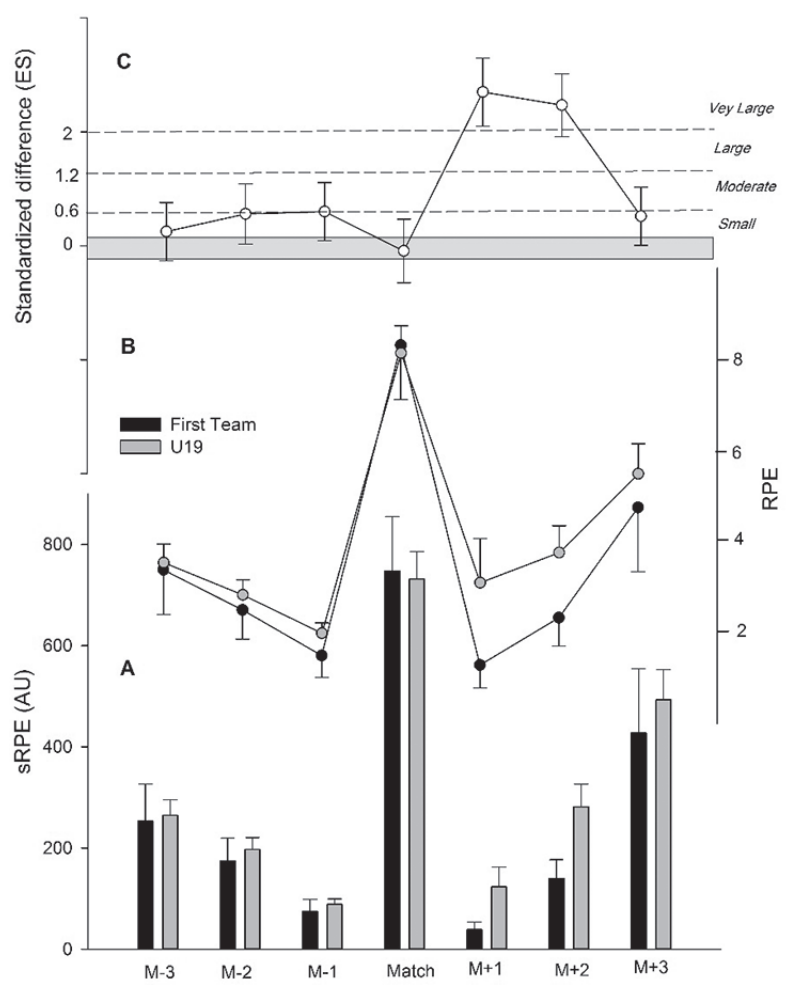

Note: ES - effect size; $30-15_{\mathrm{IFT}}-30-15$ Intermittent Fitness Test; $\mathrm{V}_{\mathrm{IFT}}-$ maximal velocity reached during the last stage of 30-15 $5_{\mathrm{IFT}}$; sRPE - session RPE; RPE - rate of perceived exertion; $\mathrm{AU}$ - arbitrary units.

Figure 2. Training load differences between the groups expressed as A) absolute differences between the groups in SRPE or B) absolute differences between the groups in RPE. Part C also shows the standardized differences $(90 \%$ confidence intervals) in SRPE between the groups. Shaded area refers to the range of trivial differences. 


\section{Discussion and conclusions}

The aim of the present study was to compare the fitness profiles and internal training loads of senior team and academy team soccer players during in-season. We hypothesized that the senior team players would have better fitness profiles and would be able to tolerate a greater amount of daily internal training loads than the academy players. Although the senior players had small to moderate levels of superiority in acceleration, maximal sprint, and COD abilities, the U19 team players were slightly better in terms of high-intensity intermittent running performance. The analyses of internal training loads showed that the U19 players tolerated small to very large higher levels of sRPE in training days. However, there was an unclear trivial difference in the internal training load on the match day between the groups.

Our results show that the senior team players have better fitness characteristics in maximal sprint, acceleration, and COD abilities. These findings are consistent with results from previous studies that showed superior speed or COD ability among older and/or higher-level players (Al Haddad, et al., 2015; Drozd, et al., 2017; le Gall, et al., 2010; Mujika, et al., 2009; Wong \& Wong, 2009). Having greater neuromuscular fitness to receive and pass the ball in a short moment, to shoot the ball strongly, to quickly dribble through opponents, to change pace immediately, and, in general, to be a winner in duels are essential needs in today's soccer, as the pace of matches is faster than in a previous decade (Barnes, et al., 2014). While it has been reported that more mature or higher-level players have a greater ability to perform high-intensity intermittent running (Bangsbo, et al., 2008; Buchheit, 2010; Mujika, et al., 2009), our results showed the opposite. It was surprising that the senior team professional players covered shorter distances than the academy players in the high-intensity intermittent running assessment. It might indicate a potential accumulated fatigue or insufficient recovery in this aspect among senior players during the first half of the competitive season.

Probably, the moderately lower level of received internal training load during in-season among the senior team players may be responsible for such a surprising result. The dose-response relationship between internal training load and changes in aerobic fitness have been reported in literature (Castagna, Impellizzeri, Chaouachi, Bordon, \& Manzi, 2011; Castagna, Impellizzeri, Chaouachi, \& Manzi, 2013; Jaspers, et al., 2016) and, more specifically, Campos-Vazquez et al. (2017) have recently shown that accumulated SRPE is a major determinant of high-intensity intermittent fitness changes among professional players. The senior team players showed better neuromuscular fitness in acceleration, maximal sprint, and COD abilities, regardless of the lower received internal training load in our study and of the inconsistent results of literature in terms of the role of accumulated loads in neuromuscular fitness change (Jaspers, et al., 2016). Less trainability of speed-related traits than of aerobic fitness traits is proposed as an explanation. Furthermore, the higher values of speedrelated fitness observed in the senior team players in our study were in line with previous reports (A1 Haddad, et al., 2015; le Gall, et al., 2010; Mujika, et al., 2009), which may be associated with possible coaches' special talent identification strategies and with the greater chances of faster players for being selected to play at the highest level.

Interestingly, similar SRPE values were recorded for both groups on the match day. This shows that soccer match play demands players to perform maximally; whether the player has a strong or poor fitness profile, he may have to perform to his full potential. Very large differences were observed in training load on $\mathrm{M}+1$ and $\mathrm{M}+2$. This may be related to a longer recovery time that the players with less high-intensity intermittent running fitness needed than their fitter counterparts (i.e., senior vs. U19 team players) (Rabbani \& Buchheit, 2016). Whether coaches have concerns about implementing more intense training or whether senior team players try to avoid higher intensities during post-match sessions, is not clear. More research is needed to further clarify this scenario.

Therefore, it seems that the lower high-intensity running fitness profiles of senior team players in the Persian Gulf League of Iran is associated with the implementation of less intense training plans. The results of the present study suggest that increasing the training load or improving the recovery strategy during the first half of a competitive year is desirable among professional players. Implementing high-intensity interval training is a special training method that has been shown to improve the highintensity intermittent performance of athletes (Buchheit \& Laursen, 2013; Buchheit \& Rabbani, 2014) and it can be implemented during in-season (Dupont, Akakpo, \& Berthoin, 2004).

As with any study, our research has some limitations. The external load was not monitored to control for physical impact of the training process on the players' responses. Despite that, our approach revealed important findings for soccer practitioners. Strength and conditioning coaches of senior teams should be aware of the importance of developing optimal high-intensity fitness levels to ensure that players are ready to optimize their performance and responses to physical demands of training and matches.

The results of the present study showed that the senior team players were moderately faster than the junior academy players. The COD ability of senior team players was also better than that of 
their younger peers, with a small effect size. The youth players, however, had a small better highintensity intermittent fitness profiles than the senior team players. Although senior team players may have stronger fitness profile in all factors than their younger counterparts in many professional clubs, this was not the case in the present study. The perceived internal training load of youth players was higher during almost all training days in the first half of a competitive season, which may be responsible for the weak high-intensity intermittent fitness profiles of senior team players in this study (Campos-Vazquez, et al., 2017). Therefore, strength and conditioning soccer coaches are encouraged to increase training intensity for senior players to enable them to reach a desirable level of perceived training load and to have better high-intensity intermittent fitness profiles.

\section{References}

Al Haddad, H., Simpson, B.M., Buchheit, M., Di Salvo, V., \& Mendez-Villanueva, A. (2015). Peak match speed and maximal sprinting speed in young soccer players: Effect of age and playing position. International Journal of Sports Physiology and Performance, 10(7), 888-896.

Bangsbo, J. (1994). The physiology of soccer-With special reference to intense intermittent exercise. Acta Physiologica Scandinavica. Supplementum, 619, 1-155.

Bangsbo, J., Iaia, F.M., \& Krustrup, P. (2008). The Yo-Yo intermittent recovery test. Sports Medicine, 38(1), $37-51$.

Barnes, C., Archer, D., Hogg, B., Bush, M., \& Bradley, P. (2014). The evolution of physical and technical performance parameters in the English Premier League. International Journal of Sports Medicine, 35(13), 1095-1100.

Buchheit, M. (2008). The 30-15 Intermittent Fitness Test: Accuracy for individualizing interval training of young intermittent sport players. Journal of Strength and Conditioning Research, 22(2), 365-374.

Buchheit, M. (2010). The 30-15 Intermittent Fitness Test: 10 year review. Myorobie Journal, 1(1), 1-9.

Buchheit, M., \& Laursen, P.B. (2013). High-intensity interval training, solutions to the programming puzzle-Part I. Sports Medicine, 43(5), 313-338.

Buchheit, M., \& Rabbani, A. (2014). The 30-15 Intermittent Fitness Test versus the Yo-Yo Intermittent Recovery Test Level 1: Relationship and sensitivity to training. International Journal of Sports Physiology and Performance, $9(3), 522-524$.

Campos-Vazquez, M.A., Toscano-Bendala, F.J., Mora-Ferrera, J.C., \& Suarez-Arrones, L.J. (2017). Relationship between internal load indicators and changes on intermittent performance after the preseason in professional soccer players. The Journal of Strength and Conditioning Research, 31(6), 1477-1485.

Castagna, C., Impellizzeri, F.M., Chaouachi, A., Bordon, C., \& Manzi, V. (2011). Effect of training intensity distribution on aerobic fitness variables in elite soccer players: A case study. The Journal of Strength and Conditioning Research, 25(1), 66-71.

Castagna, C., Impellizzeri, F.M., Chaouachi, A., \& Manzi, V. (2013). Preseason variations in aerobic fitness and performance in elite-standard soccer players: A team study. The Journal of Strength and Conditioning Research, 27(11), 2959-2965.

Djaoui, L., Chamari, K., Owen, A.L., \& Dellal, A. (2017). Maximal sprinting speed of elite soccer players during training and matches. The Journal of Strength and Conditioning Research, 31(6), 1509-1517.

Draper, J., \& Lancaster, M. (1985). The 505 test: A test for agility in the horizontal plane. Australian Journal of Science and Medicine in Sport, 17(1), 15-18.

Drozd, M., Krzysztofik, M., Nawrocka, M., Krawczyk, M., Kotuła, K., Langer, A., \& Maszczyk, A. (2017). Analysis of the 30-m running speed test results in soccer players in third soccer leagues. Turkish Journal of Kinesiology, $3(1), 1-5$.

Dupont, G.G., Akakpo, K., \& Berthoin, S. (2004). The effect of in-season, high-intensity interval training in soccer players. The Journal of Strength and Conditioning Research, 18(3), 584-589.

Helgerud, J., Engen, L.C., Wisloff, U., \& Hoff, J. (2001). Aerobic endurance training improves soccer performance. Medicine and Science in Sports and Exercise, 33(11), 1925-1931.

Hill-Haas, S.V., Dawson, B., Impellizzeri, F.M., \& Coutts, A.J. (2011). Physiology of small-sided games training in football. Sports Medicine, 41(3), 199-220.

Hopkins, W., Marshall, S., Batterham, A., \& Hanin, J. (2009). Progressive statistics for studies in sports medicine and exercise science. Medicine and Science in Sports and Exercise, 41(1), 3-13.

Impellizzeri, F.M., Rampinini, E., Coutts, A.J., Sassi, A., \& Marcora, S.M. (2004). Use of RPE-based training load in soccer. Medicine and Science in Sports and Exercise, 36(6), 1042-1047.

Jaspers, A., Brink, M.S., Probst, S.G., Frencken, W.G., \& Helsen, W.F. (2016). Relationships between training load indicators and training outcomes in professional soccer. Sports Medicine, 47(3), 533-544. 
Le Gall, F., Carling, C., Williams, M., \& Reilly, T. (2010). Anthropometric and fitness characteristics of international, professional and amateur male graduate soccer players from an elite youth academy. Journal of Science and Medicine in Sport, 13(1), 90-95.

Malone, S., Owen, A., Mendes, B., Hughes, B., Collins, K., \& Gabbett, T.J. (2017). High-speed running and sprinting as an injury risk factor in soccer: Can well-developed physical qualities reduce the risk? Journal of Science and Medicine in Sport, 21(3), 257-262.

McCall, A., Carling, C., Nedelec, M., Davison, M., Le Gall, F., Berthoin, S., \& Dupont, G. (2014). Risk factors, testing and preventative strategies for non-contact injuries in professional football: Current perceptions and practices of 44 teams from various premier leagues. British Journal of Sports Medicine, 48(18), 1352-1357.

Metaxas, T.I., Koutlianos, N., Sendelides, T., \& Mandroukas, A. (2009). Preseason physiological profile of soccer and basketball players in different divisions. The Journal of Strength and Conditioning Research, 23(6), 1704-1713.

Mujika, I., Santisteban, J., Impellizzeri, F.M., \& Castagna, C. (2009). Fitness determinants of success in men's and women's football. Journal of Sports Sciences, 27(2), 107-114.

Paul, D.J., \& Nassis, G.P. (2015). Physical fitness testing in youth soccer: Issues and considerations regarding reliability, validity, and sensitivity. Pediatric Exercise Science, 27(3), 301-313.

Rabbani, A., \& Buchheit, M. (2016). Ground travel-induced impairment of wellness is associated with fitness and travel distance in young soccer players. Kinesiology, 48(2), 200-206.

Winter, E.M., \& Maughan, R.J. (2009). Requirements for ethics approvals. Journal of Sports Sciences, $27(10), 985-985$.

Wong, D.P., \& Wong, S. (2009). Physiological profile of Asian elite youth soccer players. Journal of Strength and Conditioning Research, 23(5), 1383-1390.

Submitted: April 27, 2018

Accepted: December 7, 2020

Published Online First: April 16, 2021

Correspondence to:

Alireza Rabbani, Ph.D.

Department of Exercise Physiology,

Faculty of Sport Sciences University of Isfahan, Iran

Azadi Square, Isfahan, 817467344

Phone: +98-31-37932571

E-mail: alireza.rabbani@gmail.com

\section{Acknowledgements}

We would like to thank soccer head coach Saeid Jahangiri, who helped in the data collection of the U19 team. 